\title{
Development of a computer-generated model for the coronary arterial tree based on multislice $\mathrm{CT}$ and morphometric data
}

\author{
George S.K. Fung ${ }^{* a, b}$, W. Paul Segars ${ }^{\mathrm{b}}$, Katsuyuki Taguchib ${ }^{\mathrm{b}}$, Elliot K. Fishman ${ }^{\mathrm{b}}$, Benjamin M.W. Tsui ${ }^{\mathrm{b}}$ \\ ${ }^{a}$ Department of Electrical and Electronic Engineering, University of Hong Kong, Pokfulam Rd, Hong Kong \\ ${ }^{\mathrm{b}}$ The Russell H. Morgan Department of Radiology and Radiological Science, Johns Hopkins University, \\ Baltimore, MD 21287, USA
}

\begin{abstract}
A detailed four-dimensional model of the coronary artery tree has great potential in a wide variety of applications especially in biomedical imaging. We developed a computer generated three-dimensional model for the coronary arterial tree based on two datasets: (1) gated multi-slice computed tomography (MSCT) angiographic data obtained from a normal human subject and (2) statistical morphometric data obtained from porcine hearts. The main coronary arteries and heart structures were segmented from the MSCT data to define the initial segments of the vasculature and geometrical details of the boundaries. An iterative rule-based computer generation algorithm was then developed to extend the coronary artery tree beyond the initial segmented branches. The algorithm was governed by the following factors: (1) the statistical morphometric measurements of the connectivities, lengths, and diameters of the arterial segments, (2) repelling forces from other segments and boundaries, and (3) optimality principles to minimize the drag force at each bifurcation in the generated tree. Using this algorithm, the segmented coronary artery tree from the MSCT data was optimally extended to create a 3D computational model of the largest six orders of the coronary arterial tree. The new method for generating the 3D model is effective in imposing the constraints of anatomical and physiological characteristics of coronary vasculature. When combined with the 4D NCAT phantom, a computer model for the human anatomy and cardiac and respiratory motions, the new model will provide a unique tool to study cardiovascular characteristics and diseases through direct and medical imaging simulation studies.
\end{abstract}

Keywords: Computer-generated model, phantom, coronary artery, multislice computed tomography

\section{INTRODUCTION}

A detailed 4D computer generated model of the coronary artery tree has great potential in a wide variety of applications especially in biomedical imaging. Examples include the development and evaluation of instrumentation, and image reconstruction and processing methods for cardiovascular imaging techniques. Also, in combination with a beating heart model, it provides a useful tool in the study of coronary circulation and in the understanding of cardiovascular diseases in the future.

In medical imaging research, the 4D NURBS-based cardiac-torso (NCAT) phantom has been developed to provide a realistic and flexible mathematical model of the human anatomy and physiology [1,2]. Nonuniform rational b-spline (NURBS) [3] surfaces were employed to describe the organ shapes in the phantom. It includes a simple model for the largest branches of the coronary arteries based on 3D angiogram data. Further enhancements are required, however, in order to incorporate a more realistic and complete model of the entire coronary arterial tree.

Recently, the emergence of gated multi-slice computed tomography (MSCT) technique provides unprecedented high-resolution 4D anatomical images of the beating heart. However, the state-of-the-art imaging technique is capable of providing the delineation of only the largest 2-3 branches of the coronary artery tree. At the same time, a number of computer algorithms for the generation of the coronary arterial tree of animal hearts were proposed [4,5]. To further improve these algorithms for modeling the coronary artery tree of a human heart, we need to utilize detailed geometry of the human heart that: (1) distinguish between myocardium and epicardium layers, and (2) initialize the tree

*Correspondence: Email: skfung@eee.hku.hk; sfung2@jhmi.edu

Medical Imaging 2006: Physics of Medical Imaging, edited by Michael J. Flynn, Jiang Hsieh, Proceedings of SPIE Vol. 6142, 61421M, (2006) · 1605-7422/06/\$15 - doi: 10.1117/12.653491 
generation from segmented branches of the human coronary arterial tree. During the course of generation, the fluid mechanical constraints and optimality of the branching angles at bifurcations are met.

In this paper, we combined segmentation with a mathematical model and algorithm to develop a computer generated 3D model for the coronary arterial tree based on two datasets: (1) gated MSCT angiographic image data obtained from a normal human subject and (2) statistical morphometric data of porcine hearts. Our aim is to develop a realistic 3D model of the coronary arterial tree which satisfies the anatomical and physiological constraints of the human coronary vasculature.

\section{METHODS}

\subsection{Anatomical and Statistical Modeling of Heart and Coronary Arterial Tree}

In order to generate a realistic coronary arterial tree, both anatomical and morphometric data of the heart and coronary artery are essential. State-of-the-art MSCT cardiac data on a normal human subject provides high-resolution anatomical details of the heart and coronary arterial tree. To extend the segmented vasculature, morphometric data of porcine coronary arterial tree are used to provide the important statistical information, including lengths, diameters, and connectivities of the artery segments, of the three main coronary arteries.

\subsubsection{Anatomical Cardiac MSCT Data}

High-resolution MSCT coronary angiographic image data of a contrast enhanced normal human heart was used to define the initial segments of the coronary arteries and the geometrical details of the cardiac layers. The data were obtained from Elliot K. Fishman, M.D. of the Department of Radiology at the Johns Hopkins Medical Institutions. Figure 1 shows sample images of the MSCT dataset. They consist of 512x512 transaxial CT images of the entire heart taken at $0.6 \mathrm{~mm}$ slice thickness and a pixel size of $0.39 \mathrm{~mm}$. Only the end-diastolic phase image data, which corresponds to $0 \%$ of R-R relative delay, were used. The main coronary arteries, including left anterior descending (LAD), left circumflex artery (LCX), and right coronary artery (RCA), that were visible in the image data were manually segmented to define the initial segments. The heart layers including the epicardium and myocardium were also manually segmented to define the geometric boundaries of the vasculature. Three-dimensional NURBS surfaces were fit to the segmented structures of coronary arteries and heart layers as shown in Figure 2.
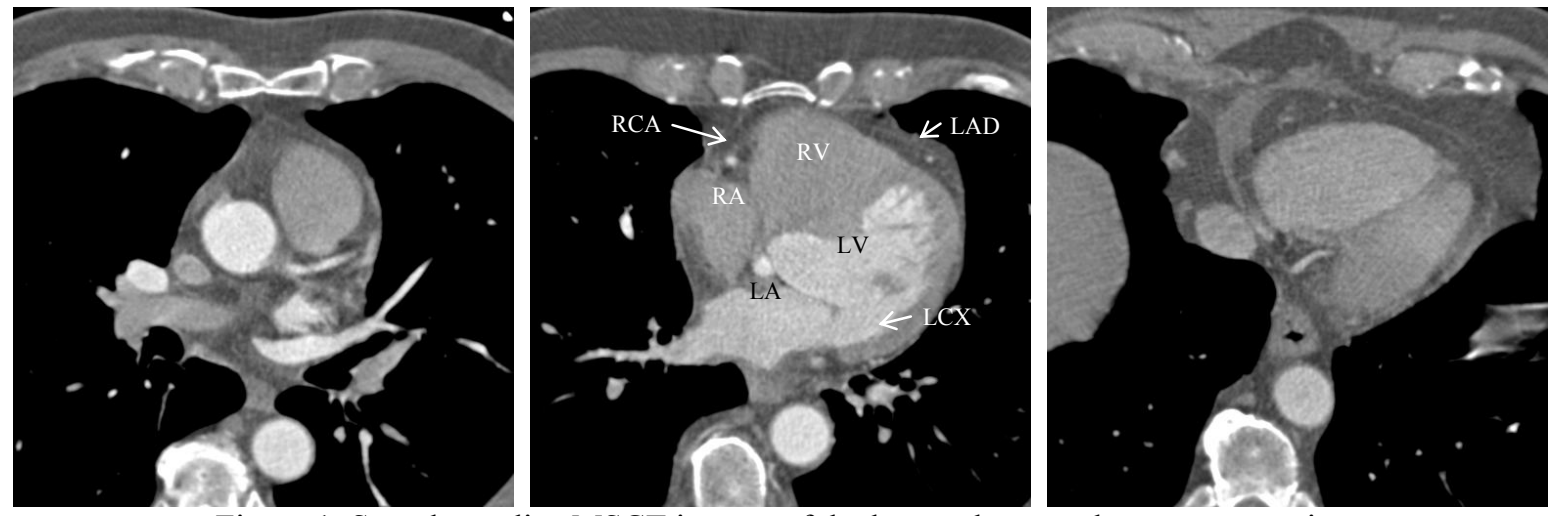

Figure 1. Sample cardiac MSCT images of the human heart and coronary arteries 

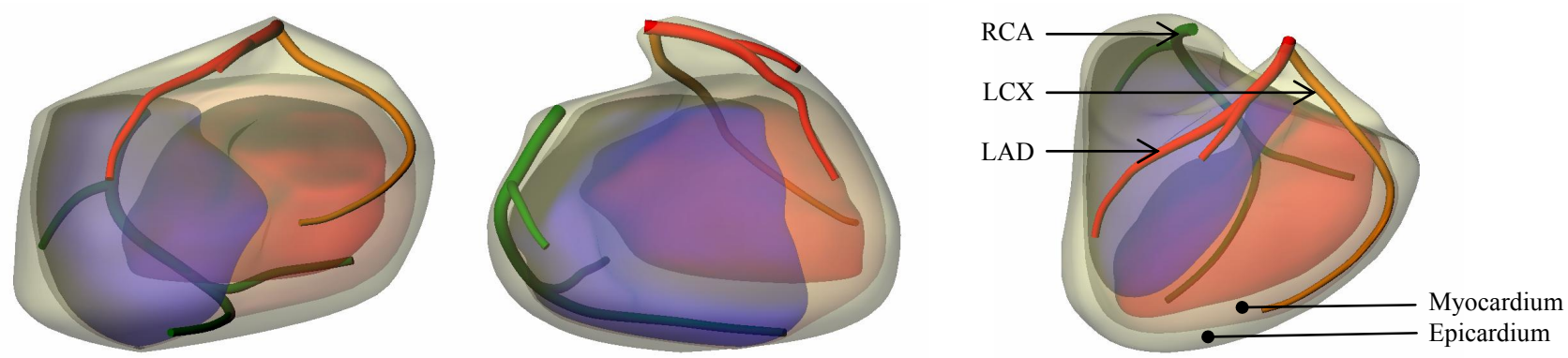

Figure 2. The segmented coronary arteries (LAD, LCX, RCA) and heart layers (myocardium and epicardium) from different views

\subsubsection{Morphometry of Coronary Arterial Tree}

The statistical morphometric measurements, including the connectivities, lengths and diameters, of each order of the coronary arterial segments from excised porcine hearts $[4,5,6]$, were used to provide the statistical guidelines for the coronary arterial tree generation. The hearts were excised and prepared with the silicone elastomer-casting method as described in [6]. The measurements were obtained from histological specimens by optical sectioning or from vascular cast. Kassab et al [6] proposed a diameter-defined Strahler ordering scheme to systematically define the orders or generations of coronary artery segments in which the arterial segments were categorized into non-overlapping diameter ranges, from the largest artery, order 11 or 10, to the smallest precapillary arterioles, order 1. According to their findings on porcine hearts, the branching of the arterial tree was dominated by bifurcations and only a very small number of branches had trifurcation, arcading or anastomese. It is reasonable to approximate the branching of the coronary arterial tree solely based on bifurcations. A total of 11 orders of vessels lied between the coronary capillaries and the aortic sinus for RCA and LAD and 10 orders for LCX.

In Table 1, the morphometric measurements of the LAD branch of the coronary artery tree are shown as an example. For each parent segment of the artery tree, our generation algorithm generated two daughter segments. The connectivity, $\mathbf{C}(m, n)$, in morphometric data was used to calculate the probability of a parent segment of order $m$ having a daughter segment of order $n$. Thus, the order of one of the two daughter segments at a bifurcation could be stochastically determined based on the connectivity probability distribution. Given the order of the daughter segment, the corresponding diameter and length were chosen from a Gaussian distribution as defined in the Table 1. Since the morphometric measurements of Kassab's paper were based on porcine hearts, the diameters and the lengths of vessel segment were required to be linearly scaled to the normal human coronary arteries as defined by Dodge Jr. et al. [7] before applying to human coronary arterial tree generation.

Table 1. The morphometric statistics of the LAD of the coronary arterial tree $[4,5,6]$

\begin{tabular}{ccccccc}
\hline & \multicolumn{7}{c}{ Parent vessel order $(\boldsymbol{m})$} \\
\cline { 2 - 7 } & $\mathbf{1 1}$ & $\mathbf{1 0}$ & $\mathbf{9}$ & $\boldsymbol{8}$ & $\mathbf{7}$ & $\mathbf{6}$ \\
\hline Diameter $(\boldsymbol{\mu m})$ & $3,176 \pm 654$ & $1,492 \pm 365$ & $715 \pm 130$ & $476 \pm 56.1$ & $303 \pm 54.5$ & $150 \pm 35.8$ \\
\hline Length $(\boldsymbol{m m})$ & $2.82 \pm 1.96$ & $2.26 \pm 1.56$ & $1.54 \pm 1.25$ & $1.09 \pm 0.83$ & $0.920 \pm 0.79$ & $0.609 \pm 0.48$ \\
\hline $\begin{array}{c}\text { Daughter vessel } \\
\text { order }(\boldsymbol{n})\end{array}$ & & & Connectivity probability $\boldsymbol{C}(\boldsymbol{m}, \boldsymbol{n})$ & & \\
\hline $\mathbf{1 0}$ & 0.188 & 0.050 & & & & \\
$\mathbf{9}$ & 0.563 & 0.191 & 0.061 & & & \\
$\mathbf{8}$ & 0.125 & 0.334 & 0.172 & 0.031 & & \\
$\mathbf{7}$ & 0.125 & 0.268 & 0.359 & 0.084 & 0.058 & \\
$\mathbf{6}$ & & 0.157 & 0.408 & 0.885 & 0.942 & \\
\hline
\end{tabular}




\subsection{Mathematical Algorithm to Extend the Coronary Arterial Tree Model}

\subsubsection{Fluid Dynamic Bifurcation Constraints}

[8] as

The relationship between the flow rate through and diameter of a branch segment was first proposed by Murray

$$
Q=C d^{n}
$$

where $Q$ is the flow rate, $C$ is a constant that depends on the organ and the fluid, $d$ is the diameter, and $n$ is a constant called the diameter exponent. Under the assumption that blood flow obeys Poiseuille's Law, Murray's Law suggested that for arterial networks minimizing pumping power, the volumetric flow rate in an arterial segment is proportional to the cube of the diameter, i.e., $n=3$. Changizi et al. [10] suggested that the flow in human coronary arteries is not entirely steady and laminar. Instead of a constant value $n$, they argued that values of $n$ should be considered within the range $[2,3]$, i.e. $n \in[2,3]$.

$d_{2}$

As shown in Figure 3, for a bifurcation site, a parent branch of diameter $d_{0}$ has two daughter branches of $d_{l}$ and

$$
d_{0}{ }^{n}=d_{1}^{n}+d_{2}{ }^{n} \text {. }
$$

Using the above relationship, Kitaoka [9] derived the equations expressing the diameters of the two daughter branches in terms of the parent diameter and the flow dividing ratio $r$ :

$$
\begin{aligned}
& d_{1}=d_{0} r^{1 / n}, \\
& d_{2}=d_{0}(1-r)^{1 / n} .
\end{aligned}
$$

The flow dividing ratio is defined as the ratio of the flow $q_{1}$ through the daughter branch receiving the smaller flow to the flow $q_{0}$ of the parent branch, i.e.

$$
r=q_{1} / q_{0},
$$

where $0<r<=0.5$. The diameter of the daughter branch with the smaller flow is less than that with greater flow, i.e. $d_{1} \leq d_{2}$.

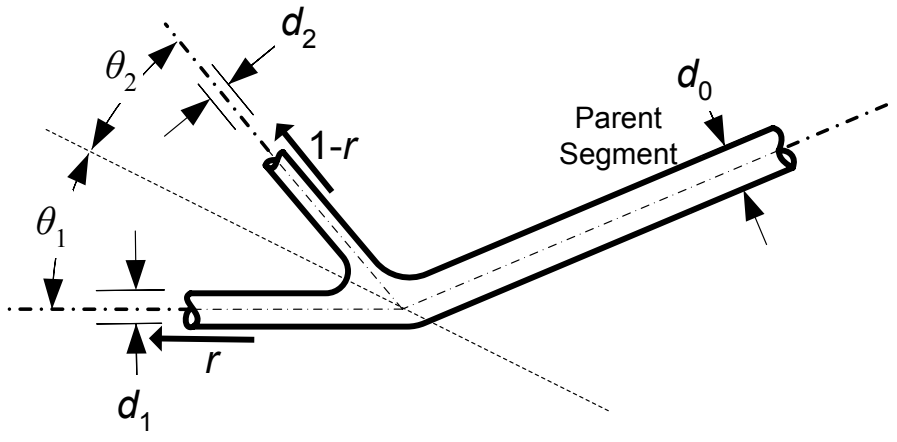

Figure 3. The relationship between flow rate, diameter and branching angle of parent and daughter vessel segments

Hacking et al. [11] suggested that drag force or shear stress acting on the lumen walls is the most likely major determinant of branch angle in vascular systems. According to Zamir's derivation [12], the optimum branching angles for minimum shear stress were

$$
\begin{aligned}
& \theta_{1}=\cos ^{-1}\left[\frac{\left(q_{0}^{2} / d_{0}^{4}\right)+\left(q_{1}^{2} / d_{1}^{4}\right)-\left(q_{2}^{2} / d_{2}^{4}\right)}{2\left(q_{0} q_{1} / d_{0}^{2} d_{1}^{2}\right)}\right], \\
& \theta_{2}=\cos ^{-1}\left[\frac{\left(q_{0}^{2} / d_{0}^{4}\right)+\left(q_{2}^{2} / d_{2}^{4}\right)-\left(q_{1}^{2} / d_{1}^{4}\right)}{2\left(q_{0} q_{1} / d_{0}^{2} d_{1}^{2}\right)}\right] .
\end{aligned}
$$


As suggested by Smith [5], as the flow through each segment at the stage of network generation was not known, $q_{1}$ and $q_{2}$ are approximated from the resistances of each segment divided by the sum of the resistances of both child segments. So, $q_{1}$ and $q_{2}$ can be approximated from the ratio of fourth power of radii of the child segments with their sum, i.e.,

$$
\begin{gathered}
q_{1}=q_{0} \frac{d_{1}{ }^{4}}{d_{1}^{4}+d_{2}{ }^{4}}, \\
q_{2}=q_{0} \frac{d_{2}{ }^{4}}{d_{1}{ }^{4}+d_{2}{ }^{4}} .
\end{gathered}
$$

By substituting eqs. (3) \& (4), and eqs. (8) \& (9) into eqs. (6) \& (7), the optimal branching angles are

$$
\begin{aligned}
& \theta_{1}=\cos ^{-1}\left[\frac{\left.\left(r^{4 / n}+(1-r)^{4 / n}\right)^{2}+(1-r)^{4 / n}-r^{4 / n}\right)}{2(1-r)^{2 / n}\left(r^{4 / n}+(1-r)^{4 / n}\right)}\right], \\
& \theta_{2}=\cos ^{-1}\left[\frac{\left.\left(r^{4 / n}+(1-r)^{4 / n}\right)^{2}+r^{4 / n}-(1-r)^{4 / n}\right)}{2 r^{2 / n}\left(r^{4 / n}+(1-r)^{4 / n}\right)}\right] .
\end{aligned}
$$

However, the two optimal branching angles were not sufficient to define the directions of daughter segments in 3D space. A branching plane, which will be defined in section 2.2.4, is required to position the daughter segments in 3D space.

\subsubsection{Self Avoidance Algorithm}

To guide the tree generation, each existing vessel segment repels the new vascular segment by growing away from its upstream position with a strength inversely related to their distance. The newly generated vasculature tended to grow away from existing structures and toward more sparsely fed layers [4].

As the segments were generated from the largest to the smallest orders, the segments of larger order or previously generated segments of equal order determined the direction of the newly generated segment. As depicted in Figure 4 , to generate a new segment at bifurcation site $\mathbf{x}_{\mathbf{c}}$, the self avoidance vector, $\mathbf{v}_{\mathbf{s}}$, was defined as

$$
\mathbf{v}_{\mathbf{s}}=\sum \frac{\left(L /\left|\mathbf{s}_{\mathbf{i}}\right|\right)^{\zeta}}{1+\left(L /\left|\mathbf{s}_{\mathbf{i}}\right|\right)^{\zeta}} \frac{\mathbf{s}_{\mathbf{i}}}{\left|\mathbf{s}_{\mathbf{i}}\right|},
$$

where $\quad \mathbf{x}_{\mathbf{i}}$ is the upstream position of all previously generated segments of the same or higher order;

$\mathbf{x}_{\mathbf{c}}$ is the upstream position of the current generating segment;

$\mathbf{s}_{\mathbf{i}}$ is the vector pointing from $\mathbf{x}_{\mathbf{i}}$ to $\mathbf{x}_{\mathbf{c}}$, i.e., $\mathbf{s}_{\mathbf{i}}=\mathbf{x}_{\mathbf{c}}-\mathbf{x}_{\mathbf{i}}$; as shown in the Figure $4, \mathbf{s}_{\mathbf{p}}$ is the one pointing

from the immediate parent position;

$\left|\mathbf{s}_{\mathbf{i}}\right|$ is the absolute distance between $\mathbf{x}_{\mathbf{i}}$ and $\mathbf{x}_{\mathbf{c}}$;

$\zeta$ is the 'avoidance exponent', governing the degree to which a new segment avoids previously generated segments, and $\zeta \approx 2$,

$L$ is the expected length of the current generating segment. 


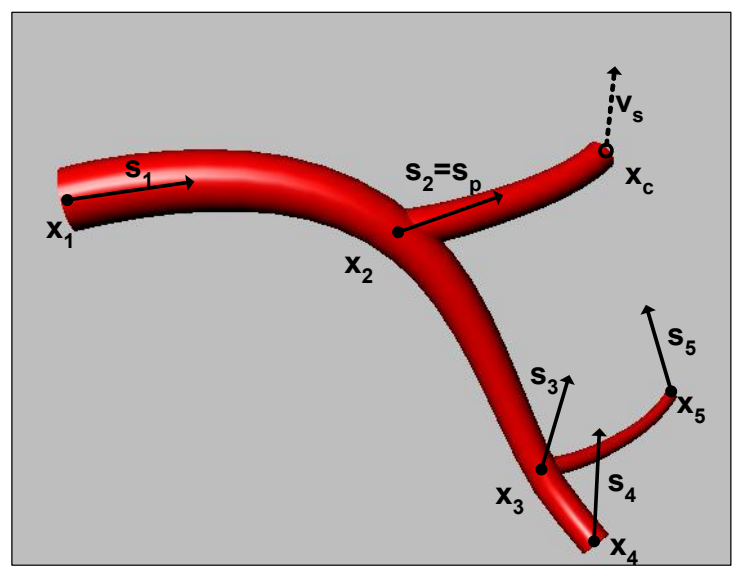

Figure 4. Self avoidance algorithm

\subsubsection{Boundary Avoidance Algorithm}

Another important factor to guide the tree generation is to avoid arterial segments to lie outside the predefined ventricle wall. The boundaries of the heart wall must constrain the vasculature within the volume defined by the ventricle surfaces. Beard et al. [4] suggested that the larger branches (order 9 or larger) should be confined to the epicardium while the smaller branches are allowed to penetrate into the myocardium.

In order to define the epicardium and myocardium layers, NURBS boundary surfaces were manually segmented from MSCT dataset. The myocardium layer is between the inner myocardial surface and the outer myocardial surface. The epicardium layer is between the outer myocardial surface and the epicardial surface. To evaluate the effect from the NURBS surface boundaries, they were approximated with many triangular surface patches as shown in Figure 5(b). The centroid, area, and normal vector were calculated for each triangular patch and the normal vectors are depicted in Figure 5(c) to (e). In Figure 5(c), the normal vectors of the inner myocardial surface are pointing outwards. The outward pointing normal vectors of outer myocardium are depicted in Figure 5(d) and the inward pointing normal vectors of outer epicardium in Figure 5(e). In order to generate the large arteries located outside of the myocardium, the larger arterial segments (order 9 and larger) were mathematically confined to grow between the epicardial surface and outer myocardial surface. For the smaller arterial segments, they were allowed to penetrate into both myocardium and epicardium layers, which were between outer inner myocardial and epicardial surfaces. To generate a new daughter segment at bifurcation site $\mathbf{x}_{\mathbf{c}}$, we defined the boundary avoidance vector, $\mathbf{v}_{\mathbf{b}}$, as,

$$
\mathbf{v}_{\mathbf{b}}=\sum_{j=1}^{N_{B}} A_{j} \mathbf{n}_{\mathbf{j}} e^{-\left(\left|\mathbf{x}_{\mathbf{c}}-\mathbf{c}_{\mathbf{j}}\right|\right) / 2 L},
$$

where $N_{B}$ is the number of boundary patches within the effective range;

$A_{j}$ is the area of the boundary patch $j$;

$\mathbf{n}_{\mathbf{j}}$ is the (outward or inward) unit normal vector for boundary patch $j$;

$\mathbf{c}_{\mathbf{j}}$ is the centroid of the boundary patch $j$;

$L$ is the expected length of the current generating segment.

In Beard at al. [4], $\mathbf{v}_{\mathbf{s}}$ and $\mathbf{v}_{\mathbf{b}}$ were directly used to define the daughter segment vector. Thus, the optimal branching angles could not be achieved. Instead, in our approach, $\mathbf{v}_{\mathbf{s}}$ and $\mathbf{v}_{\mathbf{b}}$ were used to define a branching plane which allowed the daughter branches to satisfy the optimal angles at the same time. 


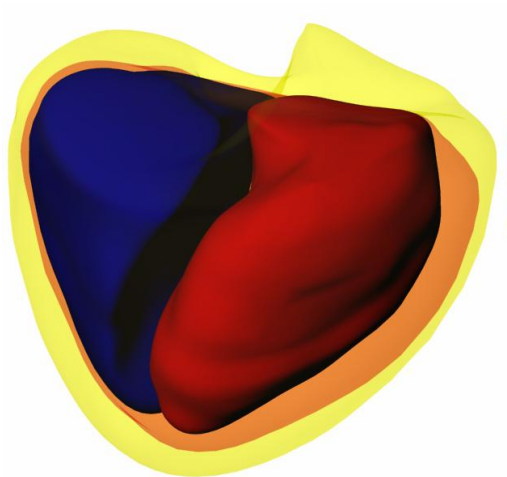

(a)

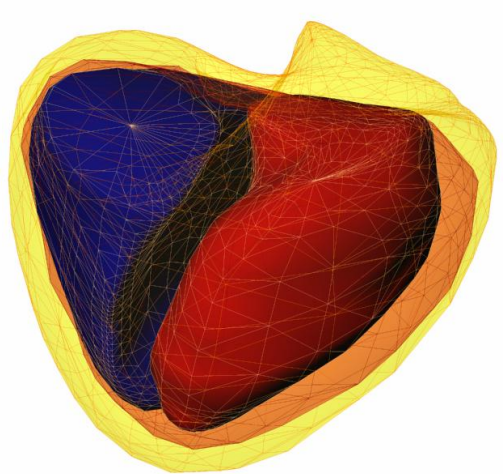

(b)

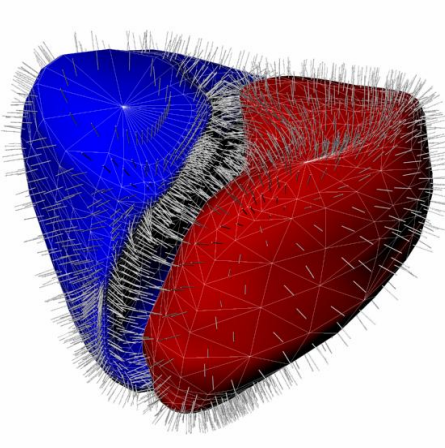

(c)

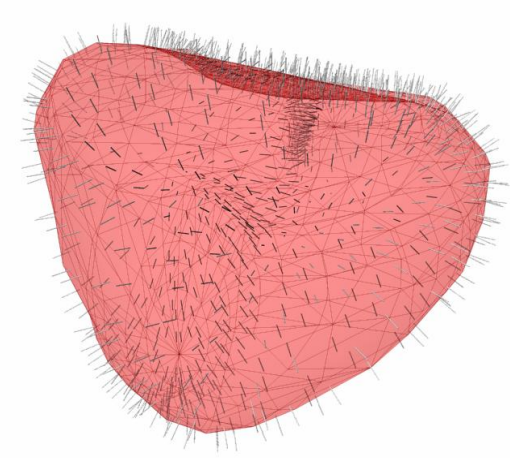

(d)

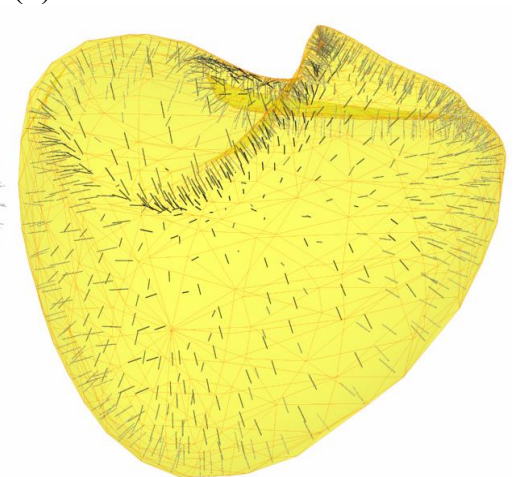

(e)

Figure 5. (a) Original NURBS surfaces; (b) Approximate triangular patch surfaces; (c) inner myocardial surface, (d) outer myocardial surface, and (e) epicardial surface.

\subsubsection{Calculation of Branching Plane and Branching Angles}

After calculating the self avoidance and the boundary avoidance vectors, they were linearly combined to form the 'combined branching vector' $\mathbf{v}_{\mathbf{d}}$ based on weighting constants $c_{s}$ and $c_{b}$, defined as

$$
\mathbf{v}_{\mathbf{d}}=c_{s} \frac{\mathbf{v}_{\mathbf{s}}}{\left|\mathbf{v}_{\mathbf{s}}\right|}+c_{b} \frac{\mathbf{v}_{\mathbf{b}}}{\left|\mathbf{v}_{\mathbf{b}}\right|},
$$

where $c_{s}+c_{b}=1$.

Since the vessel segment extended in a 3D space, a branching plane was required to confine the direction of the two daughter segments. The normal vector of the branching plane, $\mathbf{n}_{\mathbf{b}}$, was determined by cross product $\mathbf{s}_{\mathbf{p}} \times \mathbf{v}_{\mathbf{d}}$ with $\mathbf{v}_{\mathbf{d}}$, as depicted in Figure 6 and formulated as

$$
\mathbf{n}_{\mathbf{b}}=\left(\mathbf{s}_{\mathbf{p}} \times \mathbf{v}_{\mathbf{d}}\right) \times \mathbf{v}_{\mathbf{d}} .
$$

Then, $\mathbf{v}_{\mathbf{d}}$ was rotated around the axis $\mathbf{n}_{\mathbf{b}}$ by $\theta_{1}$ or $\theta_{2}$ in either clockwise or anticlockwise direction to form the daughter branch vectors $\mathbf{v}_{\mathbf{d} 1}$ and $\mathbf{v}_{\mathbf{d} 2} . \theta_{1}$ and $\theta_{2}$ were the optimum branching angles as defined in equations (10) and (11). The directions of the daughter branches were

$$
\begin{aligned}
& \text { for clockwise rotation, } \quad \mathbf{v}_{\mathbf{d} 1}=\mathbf{R}_{\mathbf{\theta}_{1}, \mathbf{n}_{\mathbf{b}}} \mathbf{v}_{\mathbf{d}} \text { and } \mathbf{v}_{\mathbf{d} 2}=\mathbf{R}_{-\boldsymbol{\theta}_{2}, \mathbf{n}_{\mathrm{b}}} \mathbf{v}_{\mathbf{d}} ; \\
& \text { or, for anticlockwise rotation, } \mathbf{v}_{\mathbf{d} \mathbf{1}}=\mathbf{R}_{-\boldsymbol{\theta}_{1}, \mathbf{n}_{\mathbf{b}}} \mathbf{v}_{\mathbf{d}} ; \text { and } \mathbf{v}_{\mathbf{d} 2}=\mathbf{R}_{\mathbf{\theta}_{2}, \mathbf{n}_{\mathbf{b}}} \mathbf{v}_{\mathbf{d}} \text {, }
\end{aligned}
$$

where $\mathbf{R}_{\mathbf{\theta}_{1}, \mathbf{n}_{\mathbf{b}}}$ was the rotation matrix which rotate the vector around the axis $\mathbf{n}_{\mathbf{b}}$ by angle $\theta_{1}$. 


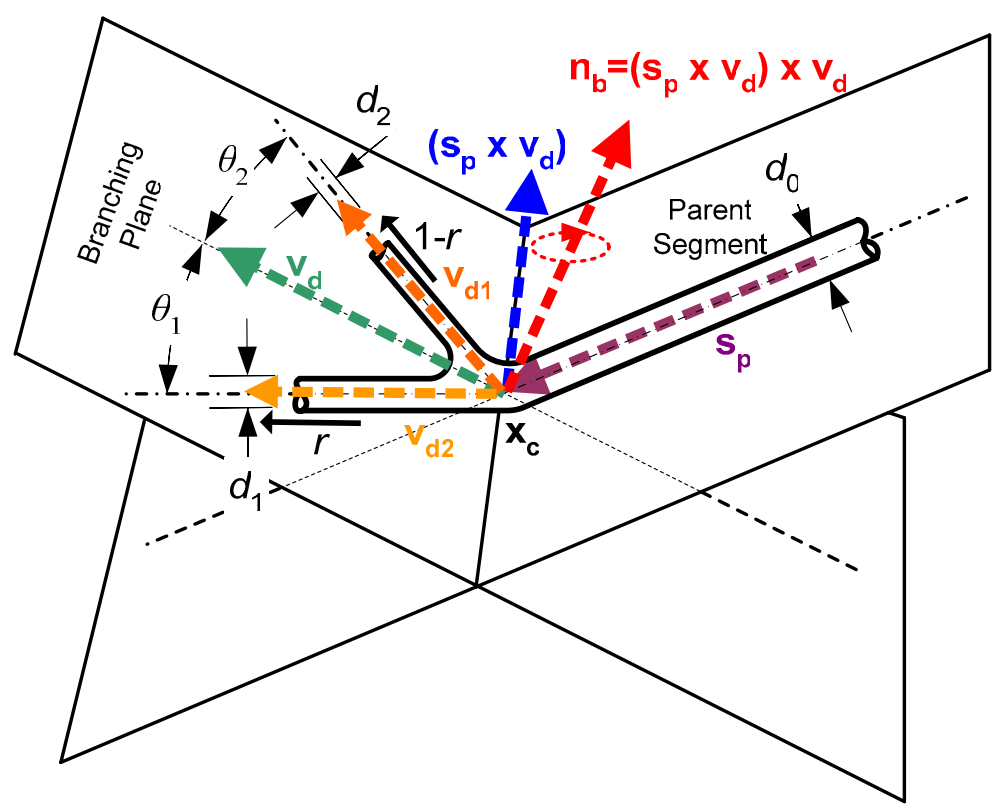

Figure 6 . The geometry of defining the branching plane and achieving optimal branching angles

The downstream point of daughter branches were calculated by adding the current bifurcation position to the daughter branch vectors and scaled by corresponding nominal lengths $L_{1}$ and $L_{2}$ which were statistically determined from the morphometric data. They were defined as

$$
\begin{aligned}
& \mathbf{x}_{\mathbf{d} \mathbf{1}}=\mathbf{x}_{\mathbf{c}}+L_{1} \mathbf{v}_{\mathbf{d} \mathbf{1}}, \\
& \mathbf{x}_{\mathbf{d} \mathbf{2}}=\mathbf{x}_{\mathbf{c}}+L_{2} \mathbf{v}_{\mathbf{d} \mathbf{2}} .
\end{aligned}
$$

Therefore, both $\mathbf{x}_{\mathbf{d} 1}$ and $\mathbf{x}_{\mathbf{d} 2}$ were on the branching plane.

\section{RESULTS AND DISCUSSION}

Similarly to our previous research in mathematically extending the airway tree in the lungs [13], nine rules were defined for producing our mathematical extension portion of the coronary arterial tree. The rules are summarized as follows:

1) two daughter branches are produced for each parent branch;

2) the daughter branches lie in the branching plane;

3) the flow rate of the parent branch is conserved after branching;

4) the diameters of the daughter branches are statistically determined by the connectivity probability and nominal diameters of the morphometric data;

5 ) the flow-dividing ratio and branching angles are found by substituting values for diameters of the daughter branches as defined in Eqs. (3), (4), (10) and (11);

6) the branch length of a given order is determined statistically by nominal length of the morphometric data;

7) the larger branches (order 9 or larger) are confined to the epicardium layer and the smaller branches are allowed to penetrate into the myocardium layer;

8) the combined branching vector is determined by the self avoidance and boundary avoidance algorithms as defined in Eq. (14); and

9) the normal vector of the branching plane is calculated from the parent branch vector and the combined branching vector as defined in Eq. (15). 
The generation algorithm successively generated two daughter segments at each bifurcation as outlined above and iterated until the segments of largest six orders were constructed. A coronary arterial tree model, including the LAD, LCX and RCA, was generated. The generated coronary arterial tree is displayed through the transparent ventricular wall in Figure 7 at different level of details. The six largest order model of the coronary arterial tree, which had diameters down to $200 \mu \mathrm{m}$, consisted of 3301, 1289, and 4069 segments for LAD, LCX, and RCA respectively.

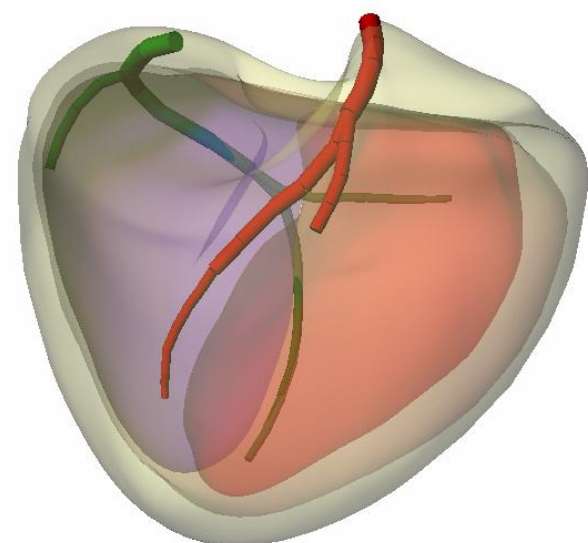

(a) Order 11

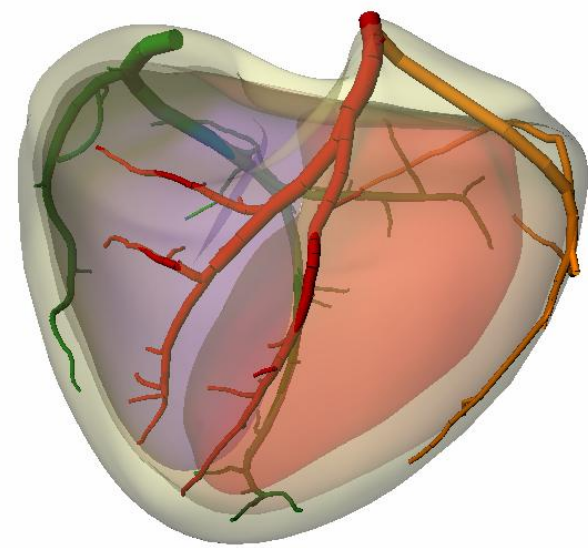

(c) Order 9 to11

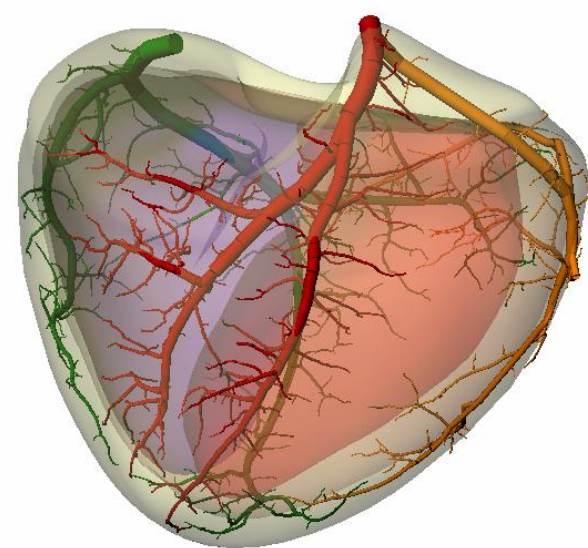

(e) Order 7 to 11

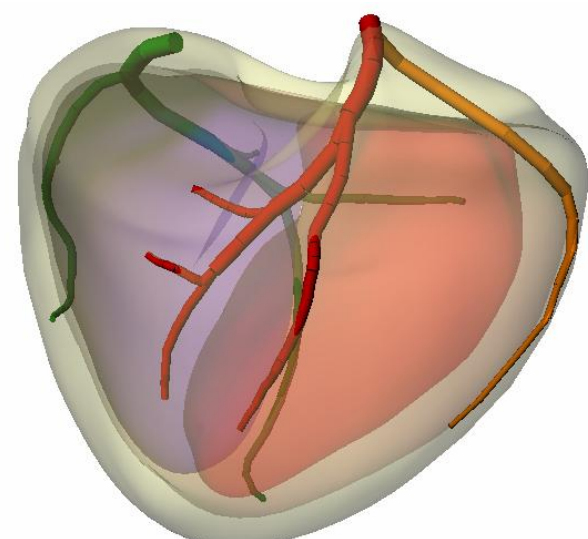

(b) Order 10 to 11

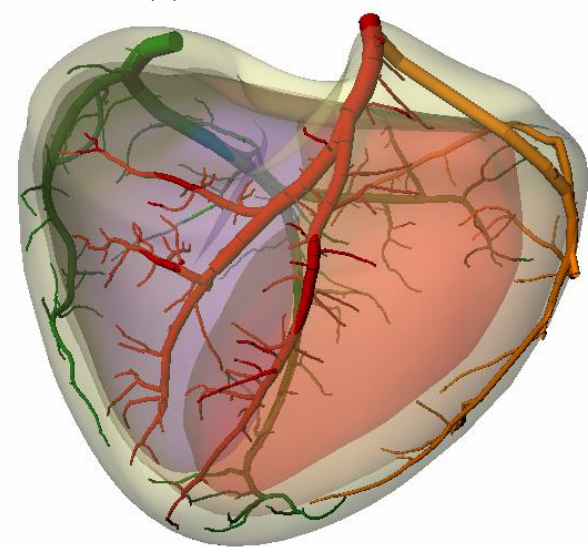

(d) Order 8 to11

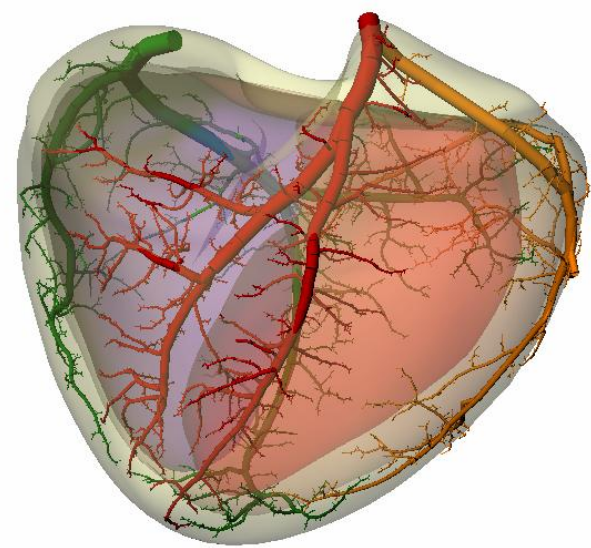

(f) Order 6 to 11

Figure 7. The computer generated coronary arterial tree at different levels of detail 


\section{CONCLUSIONS}

An anatomically realistic 3D model of the coronary arterial tree was generated by bifurcating iteratively based on a novel rule-based generation algorithm which merging the knowledge from MSCT data and statistical morphometric data. Fluid mechanic bifurcation constraints, self avoidance algorithm, boundary avoidance algorithm and formation of branching plane were employed to extend the initial segmented coronary arteries. Further work, such as uniformity of the vessel distribution in myocardium, is required to validate the anatomical accuracy of the generated vasculature model. When combined with the heart model of the 4D NCAT phantom, the new model provides a unique tool to study cardiovascular characteristics and diseases through direct and medical imaging simulation studies.

\section{ACKNOWLEDGEMENTS}

G.S.K. Fung, Ph.D. is a Visiting Scientist in the Division of Medical Imaging Physics, The Russell H. Morgan Department of Radiology and Radiological Science at the Johns Hopkins University. He is partly supported by the HKU research grant \# 10205870 and Leung Wai Sun Fellowships.

\section{REFERENCES}

1. W.P. Segars, "Development of a new dynamic NURBS-based cardiac-torso (NCAT) phantom," Ph.D. dissertation, Uni. North Carolina, 2001.

2. W.P. Segars, B.M.W. Tsui, E.C. Frey, and E.K. Fishman, "Extension of the 4D NCAT phantom to dynamic x-ray CT simulation," IEEE Nuclear Science Symposium Conference Record, pp. 3195-3199, 2003.

3. L. Piegl, "On NURBS: A survey,” IEEE Compu. Graphics Applicat., vol. 11, pp. 55-71, 1991.

4. D.A. Beard and J.B. Bassingthwaighte, "The fractal nature of myocardial blood flow emerges from a whole-organ model of arterial network," Journal of Vascular Research, vol. 37, pp. 282-296, 2000.

5. N.P. Smith, A.J. Pullan, and P.J. Hunter, "Generation of an anatomically based geometric coronary model," Annuals of Biomed. Engin., vol. 28, pp. 14-25, 2000.

6. G.S. Kassab, C.A. Rider, N.J. Tang, and Y.C.B. Fung, "Morphometry of pig coronary arterial tree," American Journal of Physiology, vol. 265, pp. H350-H365, 1993.

7. J.T. Dodge Jr., B.G. Brown, E.L. Bolson, and H.T. Dodge, "Lumen Diameter of Normal Human Coronary Arteries," Circulation, vol. 86, pp. 232-246, 1992.

8. C.D. Murray, "The physiological principle of minimum work," Proc. Nat. Acad. Sci., vol. 12, pp. 207-214, 1926.

9. H. Kitaoka, R. Takaki, and B. Suki, "A three-dimensional model of the human airway tree," J. Appl. Physiol., vol. 82, pp. 968-976, 1997.

10. M.A. Changizi, and C. Cherniak, "Modeling the large-scale geometry of human coronary arteries," Canadian Journal of Physiological Pharmacology, vol. 78, pp. 603-611, 2000.

11. W.J. Hacking, E. VanBavel, and J.A. Spaan, "Shear stress is not sufficient to control growth of vascular networks: a model study," Am. J. Physiol., vol. 270, pp. H364-H375, 1996.

12. M. Zamir, "Optimality principles in arterial branching," Journal of Theoretical Biology, vol. 62, pp. 227-251, 1976.

13. J.M. Garrity, W.P. Segars, S.B. Knisley, and B.M.W. Tsui, "Development of a dynamic model for the lung lobes and airway tree in the NCAT phantom," IEEE Transactions on Nuclear Science, vol. 50, no. 3, pp. 378-383, 2003. 